\title{
Effect of COVID-19 in Paediatric Population: Review of Recent Studies
}

Shashank Dutta ${ }^{1 *}$, Sarita Jangra Bhyan ${ }^{2}$, Ashoke Kumar Ghosh $^{3}$, Kaushal Khetwal ${ }^{4}$, Sanyam Jain $^{5}$

1. Pharm D Intern, Department of Pharmacy Practice, Teerthanker Mahaveer College of Pharmacy, TMU, Moradabad, Uttar Pradesh, India.

2. Internship Coordinator, Assistant Professor, Department of Pharmacy Practice, Teerthanker Mahaveer College of Pharmacy, TMU, Moradabad, Uttar Pradesh, India.

3. Principal, Teerthanker Mahaveer College of Pharmacy, TMU, Moradabad, Uttar Pradesh, India.

4. Pharm D Intern, Department of Pharmacy Practice, Teerthanker Mahaveer College of Pharmacy, TMU, Moradabad, Uttar Pradesh, India.

5. Pharm D Intern, Department of Pharmacy Practice, Teerthanker Mahaveer College of Pharmacy, TMU, Moradabad, Uttar Pradesh, India.

*Corresponding author's E-mail: shankydutta123@gmail.com

Received: 21-06-2020; Revised: 18-08-2020; Accepted: 25-08-2020.

DOI: $10.47583 /$ ijpsrr.2020.v64i01.015

\section{ABSTRACT}

COVID-19 is a pandemic which has entered its sixth month and is evolving in children. Besides respiratory problems, it is also known now for non-respiratory problems in children. We systematically searched the relevant articles up till June 2020. Just like in SARSCOV, MERS-COV, children are developing fewer symptoms in SARS-COV2 as compared to adults. Evidence suggests that the chances of children getting infected is same as adults but are less likely to develop severe symptoms. However, studies in various regions have shown children showing gastrointestinal symptoms, chilblain-like disease which some doctors are referring to as covid toes. MIS-C, whose pathophysiology is similar to Kawasaki syndrome. In this review, we focus on the symptoms arising in children and the treatment given to clear some of those symptoms.

Keywords: COVID-19, Pediatrics, MIS-C, Gastrointestinal symptoms, Chilblain-like lesions.

\section{INTRODUCTION}

n December, 2019 a cluster of pneumonia cases were reported in Wuhan, Hubei Province, China. The cause was later termed as Severe Acute Respiratory Syndrome-2 (SARS COV-2), a causative agent of COVID-19. Bats are considered to be the source of this new virus. On December 31, 2019, China reported the outbreak of this disease to the World Health Organization. The disease is contagious and can be transmitted by direct inhalation of infected droplets or coming in direct contact with an infected person or surface. Clinical symptoms are similar to any other acute respiratory viral infection including fever, fatigue, breathlessness, cough, sore throat. However, nasal symptoms such as sneezing or blocked nose are found to be absent in this disease.

On January30, 2020, the WHO declared the novel coronavirus outbreak a Global Health Emergency and was later declared a pandemic on March 11, 2020. As of June 23,2020 , there have been a total of 9.2 million COVID-19 cases worldwide. India has reported 449,613 cases with more than 14,000 deaths so far. About $80 \%$ of COVID-19 infected patients show mild to moderate symptoms and are easily recovered at home. In some cases, the condition worsens over time and require ventilators for breathing since the virus mainly affects the lungs. The mortality rate of this disease is low (9\%). The virus mainly harms the adults (especially geriatrics) or people with low immunity.
However, there is minimal information regarding this pandemic in children. Initially, the manifestation of COVID19 were milder in children compared to adults but now studies have linked this disease to Multisystem Inflammatory Syndrome in children (MIS-C) and gastrointestinal symptoms in children. Pediatric population is at low risk of developing severe symptoms but children suffering from other diseases like cancer, pulmonary disorder (Asthma), cardiac disease, diabetes or suppressed immune system are at a high risk of amplifying the seriousness of their condition/disease.

\section{REVIEW OF LITERATURE}

The world has been dealing with COVID-19 pandemic since the beginning of year 2020. Many studies and research are done and many are still underway. Now we can see a rise in number of new research articles based on effect, treatment and symptoms of COVID-19. Moreover, the main focus of research based on COVID-19 is adults. It has been said once and again that children are less susceptible to this disease, but later stage of this pandemic has witnessed that number of children being tested positive of COVID-19 are increasing day-by-day. Here, the authors present the review of articles based on effect of COVID-19 on pediatric population.

The age distribution of persons infected of COVID-19 is not congruent to other related disease. The three diseases, 
severe acute respiratory syndrome, Middle East respiratory syndrome, and COVID-19 are caused by corona virus and the influenza pandemic disease (H1N1 2009). The pediatric population is likely less affected of COVID-19 in comparison to adults till this time. However, the current data is encouraging but still an in-depth analysis is required to get a true knowledge of the effect of pandemic on children. ${ }^{1}$

The clinical symptoms, diagnosis, treatment, prevention, possible immune response and role of thymus in children are important factor to study. Although children are susceptible to disease caused by SARS-COV, the children of age 12 and less had shown milder disease than elder people. The studies have mentioned that children may remain as asymptomatic after getting infected with the virus and hence may act as mediator in its transmission. In many cases, SARS-COV-2 was found in feces of children, whose nose swabs and throat test were found to be negative. Neither WHO nor the US CDC has recommended any specific treatment for children. Prevention of organ failure and infections acquired in hospitals are main aim of treatment given to children. They also argued that exposure of kids to moderate coronaviruses may help them and it gave them an edge over adults. Early exposure may lead to their bodies producing antibodies and pathogens to combat COVID-19. ${ }^{2}$

Many studies can be found suggesting the slightly longer time lag between symptoms onset and day of infection (6.5-7.5 days in children compared to 5.4 days in adults). Also, SARS-COV-2 can be found in stool of children upto 30 days. Children with comorbidities and younger than 1 year should be treated with extra care. Although different treatments have been tried to fight COVID-19, none of them has proven to be more efficient than other. ${ }^{3}$ There are three factors which can help to estimate the number of children who might be severely ill due to COVID-19. First among them is the number of children at risk in population. Second factor is percentage of children at risk who will be infected. Third factor is percentage of children who will be severely ill due to COVID-19. In order to help government and policy makers especially working with pediatric medical community, one has to account for the large number of undetected cases present in the population-at-risk. ${ }^{4}$

The assessment of the children infected with novel coronavirus and their clinical profiles is important. A study was done using data of 16 children, who were 11 months to 14 years old. All considered cases were tested positive at Xiangyang Central Hospital, Hubei province, China for Covid-19 between 1 January 2020 and 17 March 2020. In Xiangyang city, most of the pediatric patients who were tested positive acquired it through their family and were often not serious. Due to lack of symptoms in children and presence of chest abnormalities, they may be diagnosed as pneumonia. We must be attentive to children as they may be asymptomatic carrier of the virus. ${ }^{5}$
The meta-analysis on characterization of COVID-19 pandemic in pediatric age group helped explore the possibility of vertical transmission from mother to neonates. The examined the breast milk in two females who were tested positive. The breast milk was tested negative and so the breast feeding is safe in this case, but a large sample size studies must be conducted to prove this. The cord blood and placenta samples were tested for new born child. However, both tested negative but it could be due to low viral load at the time of birth. Moreover, vertical transmission cannot be ruled out completely and more studies needs to be conducted to test this theory. ${ }^{6}$

A study was conducted to compare the immunological features of 12 pediatric patients with 20 adult patients. The symptoms got relieved in 3-4 days after treatment started. Furthermore, pediatric patients had mild symptoms but longer incubation period. The ones among infected pediatric patients showed different immune profile, they were found to have higher T cell amount and low inflammation. It is advised that pediatric age group population irrespective of clinical symptoms should be subjected to nucleic acid test or examination of serum IgM/IgG antibodies against the concerned virus. ${ }^{7}$

A case report of three pediatric patients was presented about the cardiovascular complications of COVID-19. The mechanism for cardiac injury and shock due to COVID-19 is still very unclear. Either it may be due to cytokine mediated myocardial inflammation or due to direct viral infection causing myocarditis. The long term follow up is needed to treat cardiac injuries resulting from COVID-19. ${ }^{8}$ The various reasons and possibilities why COVID-19 is milder in pediatric patients. This may be due to healthier respiratory tracts in pediatric patients as they have very minimum amount of exposure to hazardous cigarette and pollution smoke. The protective role of Bacillus Calmette-Guérin (BCG) vaccine to combat COVID-19 is related to heterologous immunity to others categories of pathogens which is due to phenomena of trained immunity. However it is still not proven, but trials are underway to test this hypothesis. ${ }^{9}$

\section{Covid-19 Associated MIS-C}

An observational study on 33 pediatric patients with SARSCOV2 associated multi system inflammatory syndrome (MIS-C) was conducted. The median age was 10 years with $61 \%$ being male. Fever (93\%) along with vomiting $(69 \%)$ were most common symptoms. Depressed left ventricular ejections fraction (LVEF) was found in $63 \%$ patients. Creactive protein, procalcitonin, D-dimer and pro-B-type natriuretic peptide levels were elevated in all patients. Intravenous immunoglobin was used the most for treatment (54\%). Except for one, all patients were discharged home. The children with COVID-19 associated MIS-C have a broad spectrum of severity but still requires intensive care. The recovery was almost universal and rapid. ${ }^{10}$ 
A retrospective case series was done on 35 pediatric patients who were admitted in PICU in 14 centres for severe inflammatory condition, left ventricular dysfunction and cardiogenic shock. The median age was 10 years. Inflammation markers suggested of activated macrophages and cytokine storm. 31 patients were tested positive for COVID-19. All the patients were given immune globulin and in one-third steroid therapy was added. No patient died and 25 out of 35 patients had their left ventricular function restored. MIS-C following SARS COV-2 may cause an acute cardiac decomposition in children and that immune globulin appears to be effective in the recovery of left ventricular systolic function. ${ }^{11}$ In comparison to adults, elevated inflammatory markers were less found in pediatric patients. Although new born are tested positive but none was found to be a case of vertical intrauterine transmission. Treatments suggested for children are maintaining fluids and electrolyte balances, sufficient supply of oxygen, inhalation and proper nutritional support. ${ }^{12}$

A cohort study on 6 critically ill children was done. All patients presented with severe inflammation and Kawasaki syndrome. All patients received therapy for successful treatment of Kawasaki syndrome including immunoglobulin and methylprednisolone. This therapy is found to be highly effective in reducing systemic inflammation and 5 out of 6 patients were discharged from the hospital. ${ }^{13}$ Two cases of MIS-C that lead to Kawasaki syndrome like signs in COVID-19 were reported. Both patients had increased levels of troponin which indicates myocardial injury. They received IVIG and HAT along with broad spectrum antibiotics. It is time to get more knowledge about the inflammatory syndrome which affects all organs yet whose pathophysiology is still indistinct. ${ }^{14}$

\section{Covid-19 Associated Gastrointestinal Symptoms}

Majority of COVID-19 has cases related to respiratory problems having symptoms like shortness of breath, cough and so on. However, in some regions non-respiratory symptoms in children have been seen and reported. These symptoms are related to the digestive tract such as diarrhoea along with fever, pain in the abdomen. Researchers have reported that in some cases digestive tract symptoms are observed as the first manifestation of this disease.

The study on 5 pediatric patients reported that the digestive symptoms were observed as the first manifestation. These symptoms might be related to the receptors distribution and transmission pathway associated with COVID-19 infection in humans. The virus infects people via Angiotensin Converting Enzyme 2 (ACE 2 ) receptor which can be within certain cells in the lungs as well as intestines. ${ }^{15}$ The gastrointestinal features and pathological findings of COVID-19 patients and the possibility of faecal transmission are important factors to study. The patients tested positive of COVID-19 had most common gastrointestinal symptoms as vomiting, abdominal pain, diarrhoea, anorexia, nausea and gastrointestinal bleeding. Among them diarrhoea and vomiting were most prominent in pediatric patients. More patients were reported to have gastrointestinal symptoms in later stage of pandemic in comparison to early stage. PCR tests results suggested that virus particles survived longer in the gastrointestinal tract in comparison to respiratory tract. ${ }^{16}$

A chart review of 44 children with a diagnosis of MIS-C at the children's hospital at Columbia University Irving Medical Center (CUIMC) between April 18 and May 22, 2020 showed that gastrointestinal symptoms were found to be present in $84.1 \%$ of patients. These symptoms appeared as presenting features of MIS-C. Steroids were administered to 42 patients (95.5\%) as methylprednisolone and /or hydrocortisone with no fatalities. MIS-C should be considered in patients presenting with $\mathrm{Gl}$ symptoms and have a history with SARS-COV2. ${ }^{17}$ Two confirmed pediatric cases from two family clusters had mild respiratory or gastrointestinal symptoms. Their chest CT was found normal and both patients recovered quickly after their symptomatic treatment. ${ }^{18}$ Eight children with COVID-19 had symptoms of atypical appendicitis in a single centre in UK. All eight children presented with similar symptoms including fever, diarrhoea, abdominal pain, vomiting. Except one, all patients had increased levels of C-Reactive protein. They were treated with antibiotics and fluids. In some cases, immunoglobulin and steroid treatment was provided. There was no casualty involved. ${ }^{19}$

A study with patients less than 18 years of age with condition of acute myocarditis and hypotensive shock. Their complaints were of fever, severe pain in the abdomen, vomiting. All had raised levels of CRP, procalcitonin. Some had acute renal failure but none required renal replacement therapy. All were treated with IV immunoglobulin and 2 children received corticosteroids as well. ${ }^{20} 148$ children were admitted in Wuhan, China with mild to moderate disease. Their median age was 84 months. Fever $(40.5 \%)$, cough $(44.6 \%)$, diarrhoea or vomiting (21.6\%) were among the most common symptoms. Hepatic markers were high in moderate cases as compared to mild ones but majority was within the normal range. There were no deaths and all 148 patients were discharged. ${ }^{21}$

\section{Dermatology}

Doctors have identified a new symptoms in COVID-19 patients, informally dubbed as "COVID TOES". Purple or blue lesions were noticed on patient's feet and toes. These lesions are characterized by hot burning sensation and are painful when touched. These type of lesions are mostly observed in pediatric population because of their high immune system as compared to adults (especially geriatrics). Because of good immunity, COVID-19 is not able to attack directly to the lungs. Other symptoms like lesions are observed. In some cases, the result for COVID19 was reported as negative but later these same patients 
were tested positive even after being in quarantine this whole time. Researchers have come up with two hypotheses for these symptoms. First is that these lesions are an inflammatory response to a patient's foot and toes. Secondly, it could be a clotting of blood vessels which causes pain when touched and is bluish purple in colour. Some researchers considered that the body's inflammatory response against the infection could be beneficial but then it could also lead to self-damage. According to some doctors, similar inflammatory responses were observed in cases of viral pneumonia or bird flu. So it was not really surprising for these symptoms to be noticed in COVID-19 patients.

22 children in the emergency department of a hospital in Spain were admitted. These children presented with chilblain- like lesions on their toes. Some had lesions on their fingers as well. These chilblains were in the form of violet coloured swelling and erythematous to purpuric macules on their toes, feet, hands. The etiology and pathogenesis is still unknown but is a mildly symptomatic condition with a good prognosis. These children were observed during the COVID-19 pandemic and did not require any specific therapy. There are many other studies that showed these lesion like symptoms during this pandemic in pediatric population. ${ }^{22}$ Children and adolescents are also suffering from acroischemic foot lesions, probably the expression of vascular disorders related to SARS-COV2 infection. ${ }^{23}$ There are some letters which shows that they also saw cases that can show cutaneous manifestations related to SARS-COV2 infection. ${ }^{24,25}$

\section{CONCLUSION}

COVID-19 has now posed a serious threat to the world by infecting millions of people regardless of their age, gender. Children are less susceptible to the disease than adults, the reason being their high immunity as compared to adults especially elderly people. However, cases have been reported in some regions where children are presenting not only respiratory symptoms but also non-respiratory symptoms such as gastrointestinal symptoms (diarrhoea, vomiting, abdominal pain), COVID-19 associated Multisystem Inflammatory Syndrome in children (MIS-C), chilblain-like lesions.

Gastrointestinal symptoms are caused because of the interaction between the virus and Angiotensin Converting Enzyme 2 receptor (ACE2) which is present along the intestinal wall and in all organs. Majority of patients with COVID-19 associated MIS-C had left ventricular dysfunction. They also had elevated levels of C-Reactive Protein, procalcitonin. In case of patients with lesions on their feet and hands, they had purple-blue coloured swelling and although there was no exact etiology, they were believed to be an inflammatory response or clotting of blood vessels which didn't require any specific therapy. Patients with MIS-C were mainly treated with IV immunoglobulin and steroids in some cases. Same treatment was recommended in GI patients.
There is no current vaccine available for curing SARS-COV2. However, certain drugs are now getting approval as alternatives for COVID-19. Researchers in The United Kingdom came up with Dexamethasone for patients in severe conditions. Recently CDSCO has accepted the use of favipiravir against mild to moderate cases. Remdesivir is another drug known for bringing down the viral load.

Acknowledgment: We acknowledge Teerthanker Mahaveer University for providing resources \& valuable support.

\section{REFERENCES}

1. Gupta S, Malhotra N, Gupta N, Agrawal, S and Ish P, The curious case of coronavirus disease 2019 (COVID-19) in children, The Journal of Pediatrics, 2020.

2. Rehman S, Majeed T, Ansari, MA, Ali U, Sabit $\mathrm{H}$ and AlSuhaimi EA, Current scenario of COVID-19 in pediatric age group and physiology of immune and thymus response, Saudi Journal of Biological Sciences, 2020.

3. Patel NA, Pediatric COVID-19: Systematic review of the literature, American Journal of Otolaryngology, 2020, 102573.

4. Salemi JL, Menard J and Pathak EB, Estimating severe and critical illness in children with COVID-19, Early Human Development, 2020.

5. Song W, Li J, Zou N, Guan W, Pan J and Xu W, Clinical features of pediatric patients with coronavirus disease (COVID19), Journal of Clinical Virology, 2020, 104377.

6. Mustafa NM and Selim LA, Characterisation of COVID-19 Pandemic in Paediatric Age Group: A Systematic Review, Journal of Clinical Virology, 2020, 104395.

7. Chen J, Zhang ZZ, Chen YK, Long QX, Tian WG, Deng HJ, Hu $\mathrm{JL}$, Zhang XX, Xiang JL, Wang DX and Hu P, The clinical and immunological features of pediatric COVID-19 patients in China, Genes \& Diseases, 2020.

8. Joshi K, Kaplan D, Bakar A, Jennings JF, Hayes DA, Mahajan S, Misra N, Mitchell E, Sweberg TM, Taylor MD and Capone CA, Cardiac dysfunction and shock in pediatric patients with COVID-19, JACC: Case Reports, 2020.

9. Balasubramanian S, Rao NM, Goenka A, Roderick M and Ramanan AV, Coronavirus Disease 2019 (COVID-19) in Children-What We Know So Far and What We Do Not, Indian Pediatrics, 57, 2020, 435-442.

10. Kaushik S, Aydin SI, Derespina KR, Bansal PB, Kowalsky S, Trachtman R, Gillen JK, Perez MM, Soshnick SH, Conway Jr EE and Bercow A, Multisystem Inflammatory Syndrome in Children (MIS-C) Associated with SARS-CoV-2 Infection: A Multi-institutional Study from New York City, The Journal of Pediatrics, 2020.

11. Belhadjer Z, Méot $M$, Bajolle $F$, Khraiche $D$, Legendre $A$, Abakka S, Auriau J, Grimaud M, Oualha M, Beghetti M and Wacker J, Acute heart failure in multisystem inflammatory syndrome in children (MIS-C) in the context of global SARSCoV-2 pandemic, Circulation, 2020.

12. Ludvigsson JF, Systematic review of COVID-19 in children shows milder cases and a better prognosis than adults, Acta Paediatrica, 109(6), 2020, 1088-1095. 
13. Chiotos K, Bassiri H, Behrens EM, Blatz AM, Chang J, Diorio C, Fitzgerald JC, Topjian A and John ARO, Multisystem Inflammatory Syndrome in Children during the COVID-19 pandemic: a case series, Journal of the Pediatric Infectious Diseases Society, 2020.

14. Saeed A, Sanaei A, Ghotbabai SH, Shorafa E, Case report: Pediatric patients with COVID-19 presented as multi-system inflammatory syndrome, 2020.

15. Cai X, Ma Y, Li S, Chen Y, Rong Z and Li W, Clinical Characteristics of 5 COVID-19 Cases With Non-respiratory Symptoms as the First Manifestation in Children. Frontiers in Pediatrics, 8, 2020, 258.

16. Tian $Y$, Rong L, Nian $W$ and $\mathrm{He} Y$, Gastrointestinal features in COVID-19 and the possibility of faecal transmission, Alimentary pharmacology \& therapeutics, 51(9), 2020, 843-851.

17. Miller J, Cantor A, Zachariah P, Ahn D, Martinez M and Margolis K, Gastrointestinal symptoms as a major presentation component of a novel multisystem inflammatory syndrome in children (MIS-C) that is related to COVID-19: a single center experience of 44 cases, Gastroenterology, 2020.

18. Ji LN, Chao S, Wang YJ, Li XJ, Mu XD, Lin MG and Jiang RM, Clinical features of pediatric patients with COVID-19: a report of two family cluster cases, World Journal of Pediatrics, 2020, 1-4.

19. Tullie L, Ford K, Bisharat M, Watson T, Thakkar H, Mullassery D, Giuliani S, Blackburn S, Cross K, De Coppi P and Curry J, Gastrointestinal features in children with COVID-19: an observation of varied presentation in eight children. The Lancet. Child \& Adolescent Health, 2020.

20. Grimaud M, Starck J, Levy M, Marais C, Chareyre J, Khraiche $D$, Leruez-Ville $M$, Quartier $P$, Léger $P L$, Geslain $G$ and Semaan N, Acute myocarditis and multisystem inflammatory emerging disease following SARS-CoV-2 infection in critically ill children, Annals of Intensive Care, 10(1), 2020, 1-5.

21. Wu H, Zhu H, Yuan C, Yao C, Luo W, Shen X, Wang J, Shao J and Xiang $Y$, Clinical and Immune Features of Hospitalized Pediatric Patients With Coronavirus Disease 2019 (COVID19) in Wuhan, China, JAMA Network Open, 3(6), 2020, e2010895-e2010895.

22. Andina D, Noguera-Morel L, Bascuas-Arribas M, GaiteroTristán J, Alonso-Cadenas, JA, Escalada-Pellitero S, Hernández-Martín Á, de la Torre-Espi M, Colmenero I and Torrelo A, Chilblains in children in the setting of COVID-19 pandemic. Pediatric Dermatology, 2020.

23. Mazzotta F and Troccoli T, Acute acro-ischemia in the child at the time of COVID-19. European Journal of Pediatric Dermatology, 30(2), 2020, 71-74.

24. Recalcati S, Barbagallo T, Frasin LA, Prestinari F, Cogliardi A, Provero MC, Dainese E, Vanzati A and Fantini F, Acral cutaneous lesions in the time of COVID-19. Journal of the European Academy of Dermatology and Venereology, 2020

25. Piccolo V, Neri I, Filippeschi C, Oranges T, Argenziano G, Battarra VC, Berti S, Manunza F, Belloni Fortina A, Di Lernia V and Boccaletti V, Chilblain-like lesions during COVID-19 epidemic: a preliminary study on 63 patients. Journal of the European Academy of Dermatology and Venereology, 2020.

Source of Support: None declared.

Conflict of Interest: None declared.

For any question relates to this article, please reach us at: editor@globalresearchonline.net New manuscripts for publication can be submitted at: submit@globalresearchonline.net and submit_ijpsrr@rediffmail.com 